\title{
Robustness of ventilation systems in the control of walking-induced indoor fluctuations: Method development and case study
}

\author{
Jianlin Ren ${ }^{1}$, Junjie He', Xiangfei Kong' $(\varangle)$, Hongwan Li $^{2}$ \\ 1. School of Energy and Environmental Engineering, Hebei University of Technology, Tianjin, 300401, China \\ 2. Department of Environmental Engineering Sciences, University of Florida, USA
}

\begin{abstract}
Walking-induced fluctuations have a significant influence on indoor airflow and pollutant dispersion. This study developed a method to quantify the robustness of ventilation systems in the control of walking-induced fluctuation control. Experiments were conducted in a full-scale chamber with four different kinds of ventilation systems: ceiling supply and side return (CS), ceiling supply and ceiling return (CC), side supply and ceiling return (SC), and side supply and side return (SS). The measured temperature, flow and pollutant field data was (1) denoised by FFT filtering or wavelet transform; (2) fitted by a Gaussian function; (3) feature-extracted for the range and time scale disturbance; and then (4) used to calculate the range scale and time scale robustness for different ventilation systems with dimensionless equations developed in this study. The selection processes for FFT filtering and wavelet transform, FFT filter cut-off frequency, wavelet function, and decomposition layers are also discussed, as well as the threshold for wavelet denoising, which can be adjusted accordingly if the walking frequency or sampling frequency differs from that in other studies. The results show that for the flow and pollutant fields, the use of a ventilation system can increase the range scale robustness by $19.7 \%-39.4 \%$ and $10.0 \%-38.8 \%$, respectively; and the SS system was $7.0 \%-25.7 \%$ more robust than the other three ventilation systems. However, all four kinds of ventilation systems had a very limited effect in controlling the time scale disturbance.
\end{abstract}

\author{
Keywords \\ FFT filtering; \\ wavelet denoising; \\ Gaussian fitting; \\ feature extraction; \\ robust
}

\author{
Article History \\ Received: 28 November 2021 \\ Revised: 04 January 2022 \\ Accepted: 24 January 2022 \\ ๑) Tsinghua University Press 2022
}

\section{Introduction}

The rapid spread of SARS-CoV-2 infections and the associated high morbidity during the COVID-19 pandemic have attracted significant attention worldwide (Desai et al. 2021; Dong et al. 2021; Kong et al. 2021; Liu et al. 2021a; Srivastava et al. 2021; Ren et al. 2022b). In addition to SARS-CoV-2, many infectious diseases (e.g., influenza and tuberculosis) are associated with airborne particulate matter transmission, since the pathogens are contained in respiratory particles (Wang et al. 2019; Satheesan et al. 2020; Dai and Zhao 2020; Ye et al. 2021; Liu et al. 2021b; Liu et al. 2022; Mckeen and Liao 2022). Recent studies have suggested that the wake flow generated by human walking has a significant effect on indoor flow field distribution and particle transmission (Hang et al. 2014; Liu et al. 2020; Lv et al. 2021). There are increasing concerns about walking-induced airflow and pollutant field disturbance in specific indoor environments, such as hospital wards (Liu et al. 2020), aircraft cabins (Chang et al. 2017), cleanrooms (Whyte et al. 2013) and operating theatres (Brohus et al. 2006).

In recent years, numerous investigations have used computational fluid dynamics (CFD) simulations to study the effects of human walking on flow and pollutant characteristics. Mazumdar et al. (2011) used a validated CFD model to simulate the transport of pollutants in airliner cabins. Their calculations indicated that a crew member's walking along the airliner aisle could induce the transport of a pollutant from the source to any location through which the crew member passed. Wang and Chow (2011) employed a dynamic mesh model to represent human movement. The dynamic mesh technique was also used by Liu et al. (2020), who found that a nurse's walking in an operating theatre could generate changes in the airflow

E-mail: xfkong@hebut.edu.cn 
field and redistribution of bioaerosols. In addition, Cao et al. (2017) incorporated a momentum source into the Navier-Stokes equations to simulate human walking. Their momentum theory method reduced the calculation time by up to $90 \%$, while maintaining a computational accuracy similar to that of the dynamic mesh method. Meanwhile, CFD simulations indicated that human walking-induced disturbances would have a significant effect on the indoor flow and pollutant fields. For the time scale of the disturbance, once the movement stops, it would take a few minutes for the temperature field, as well as the flow field, to return to the pre-disturbance state. In addition, the disturbance time of the pollutant field would be extended (Tao et al. 2017). For the range scale of the disturbance, the wake flow generated by human walking would accelerate the spread of pollutants (Lv et al. 2021) and expand the scale of the spread (Chang et al. 2017). However, experimental studies on walking-induced disturbances in the literature are limited (Poussou et al. 2010; Wu and Lin 2015; Liu et al. 2020), especially on the disturbance characteristics arising from a human being's walking in a full-scale chamber $(\mathrm{Wu}$ and Lin 2015). In addition, many high-frequency fluctuations have been found in experimental data on walking-induced disturbances ( $\mathrm{Wu}$ and Lin 2015), and thus it is difficult to use the data directly for CFD simulation verification. Ideally, the experimental data would be carefully denoised to further extract fluctuant features (Mallat 1989; Daubechies 1992; Bachman et al. 2012; Veer and Agarwal 2015).

Many existing studies have emphasized the effects of different ventilation systems on indoor flow field construction and contaminant removal (Ren et al. 2020; Lv et al. 2021; Ji et al. 2021; Mei et al. 2021; Ren et al. 2022a; Yin et al. 2022). However, the control effect of ventilation systems on walking-induced disturbances has seldom been investigated. Hang et al. (2014) simulated the influence of human walking on contaminant dispersion in a six-bed isolation room. They found that in scenarios in which a person walked or in the absence of human intervention, a ceiling-level exhaust ventilation system controlled airborne particle transmission more effectively than systems with floor-level exhausts. Wu and Lin (2015) studied the influence of human walking under three air-distribution modes in a full-scale chamber: stratum, displacement and mixing ventilation. Their study showed that stratum ventilation had potential for scenarios with frequent movements, although there was no quantitative method for comparing the effectiveness of various ventilation systems in the control of walking-induced flow and pollutant field disturbances.

In fact, the ventilation system was particularly important for the control of walking-induced disturbances in specific places that require a highly stable indoor environment (cleanrooms, operation rooms, isolation rooms, etc.). In cleanrooms, human walking would increase the risk of microbe-carrying particles diffusion onto the manufactured product (Whyte et al. 2013). Besides, operating rooms would have a great risk in the hygienic environment due to the infection of the surgical site in the hospital (Agirman et al. 2020). The increase in bacteria-carrying particles concentration caused by medical staff walking was an important cause of infection at the surgical site (Liu et al. 2020). Therefore, robustness (Weisberg 2006) needs to be introduced to quantify the effectiveness of a ventilation system in controlling walking-induced disturbances. In this study, the temperature, flow and pollutant field fluctuations caused by human walking under four different ventilation systems were measured in a full-scale chamber. The experimental data was first denoised by fast Fourier transform (FFT) filtering (Daubechies 1992; Bachman et al. 2012) or wavelet noise reduction (Mallat 1989; Daubechies 1992; Bachman et al. 2012; Veer and Agarwal 2015), which have been widely used in compressed images, electrocardiograms, biological cell signals and audio fields (Sifuzzaman et al. 2009; Gothwal et al. 2011; Paskaranandavadivel et al. 2013; John et al. 2020). These application methods have a certain degree of commonality, and they were filtered according to the feature frequency that needed to be extracted. Next, the denoised data was fitted by a Gaussian function (Guo 2011; Wang et al. 2013) to obtain a highly precise result. The walking-induced fluctuations under different ventilation system were then feature-extracted according to the fitted Gaussian function (Wang et al. 2013). Finally, the robustness of different ventilation systems was calculated using the normalized datasets.

In order to address the research gaps described above and provide further information about the robustness of ventilation systems in controlling human walking-induced fluctuations, this study conducted experiments in a full-scale chamber. This investigation was focused mainly on the development of data-processing methods, i.e., the use of FFT filtering, wavelet transform, Gaussian function fitting and robustness analysis. A detailed explanation of the experimental results using the methods described above will be presented in a separate study.

\section{Methodology}

\subsection{Experimental setup}

Experiments were conducted in a chamber with dimensions of $6.0 \mathrm{~m}(L) \times 5.9 \mathrm{~m}(W) \times 2.5 \mathrm{~m}(H)$, and the walkinginduced temperature, flow and pollutant field fluctuation characteristics were measured. As shown in Figure 1, there were two parallel rows of air inlets/outlets on the two 


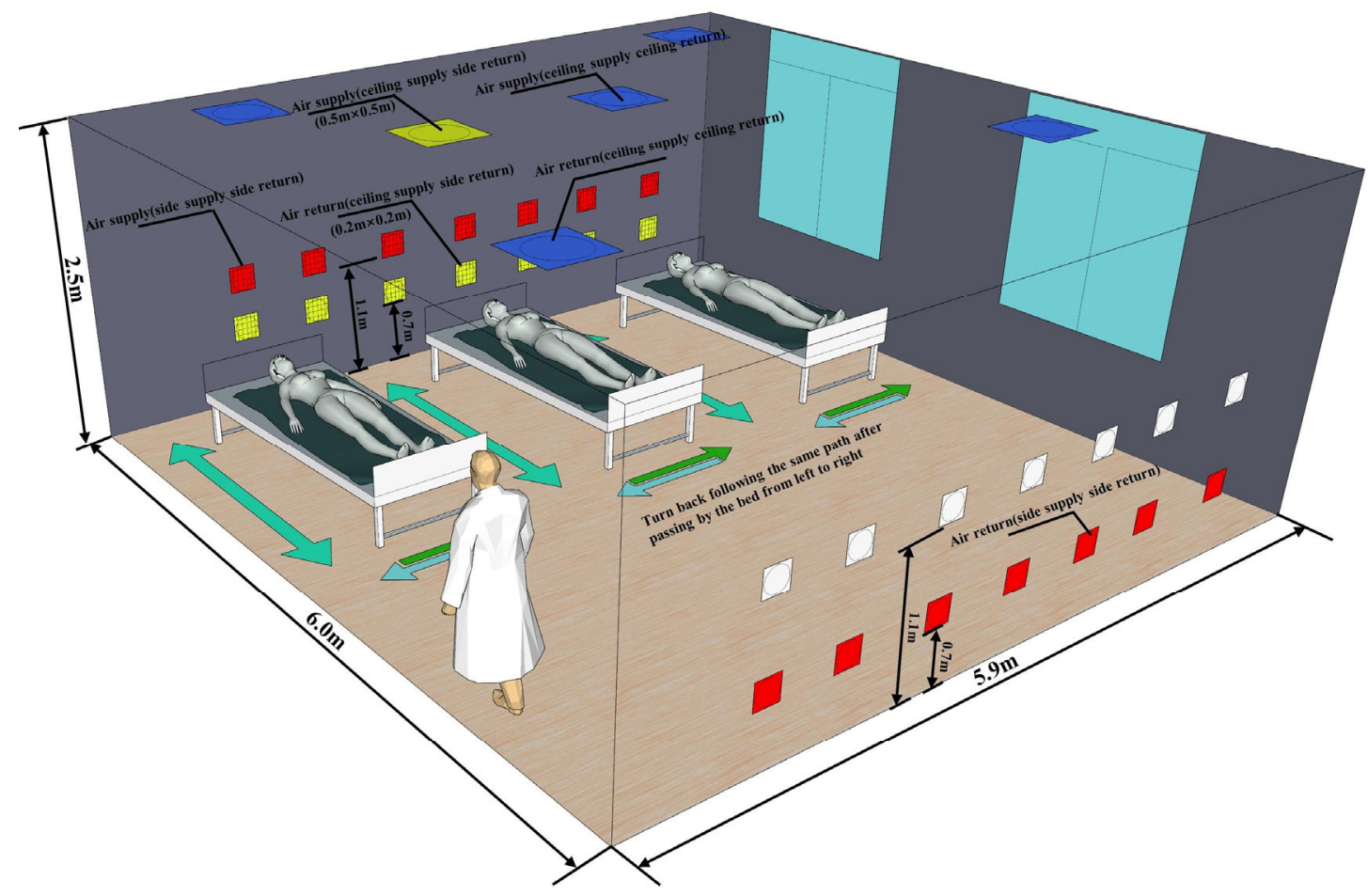

Fig. 1 Diagram of the experimental chamber

opposite side walls: one row at a height of $0.7 \mathrm{~m}$ above the ground, and the other row at $1.1 \mathrm{~m}$ above the ground. The dimensions of each diffuser on the side wall were $0.2 \mathrm{~m} \times$ $0.2 \mathrm{~m}$. There were also six diffusers on the ceiling with dimensions of $0.5 \mathrm{~m} \times 0.5 \mathrm{~m}$. Detailed information about the chamber can be found in Kong et al. (2021). In the present study, four different types of ventilation systems were evaluated: ceiling supply and side return (CS), ceiling supply and ceiling return (CC), side supply and ceiling return (SC), and side supply and side return (SS). The supply air volume was maintained at $885 \mathrm{~m}^{3} / \mathrm{h}$ (10 air exchange rate per hour), and the temperature in the chamber was consistent at $\pm 23^{\circ} \mathrm{C}$ for all ventilation systems. Three beds were placed in the chamber, and one non-heated dummy model was placed on each bed. The dummy on the left represented the particle generator, and the other two dummies represented the receivers. Prior to each experiment, the floor of the chamber was mopped at least twice to prevent the resuspension of particles from the floor by walking.

The pollutant field measurement was conducted for 70 minutes under each ventilation mode, and the particles were released at a consistent rate by an aerosol generator. The ventilation system was not turned on until 15 minutes after the start of particle release. Measurement of the temperature and flow fields began 25 minutes from the initial release of particles. At the same time, a male volunteer (who is also the author of the paper) with a height of $1.75 \mathrm{~m}$ and weight of $60 \mathrm{~kg}$ walked in the chamber at a constant speed of approximately $60 \mathrm{steps} / \mathrm{min}$. The volunteer wore personal protective equipment, consisting of a mask, examination gloves and glasses. The walking route is shown in Figure 1. The volunteer walked in the chamber for 5 minutes, repeating the process three times. The interval between walks was 10 minutes. The total sampling time of 70 minutes was determined according to many preliminary experiments to provide enough time for pollutant field to reach a stable state before walking and reconstruct after walking.

\subsection{Instruments used in the experiments}

An aerosol generator (Model 9302, TSI Inc., St. Paul, MN, USA) was used to generate polydisperse $\mathrm{NaCl}$ particles at a flow rate of $6.5 \mathrm{~L} / \mathrm{min}$. The particle concentration of the outlet of the generate was kept as 50,000 particles $/ \mathrm{cm}^{3}$ throughout the sampling. The outlet was connected to the mouth of the left-hand dummy. T-type thermocouples were used to measure the temperature in the chamber with a frequency of $0.1 \mathrm{~Hz}$. A data-acquisition instrument (Model 34972A, Aglient, Santa Clara, CA, USA) was used to record temperature data. The measurable range was $-200{ }^{\circ} \mathrm{C}$ to $260^{\circ} \mathrm{C}$, and the accuracy was $0.1{ }^{\circ} \mathrm{C}$. As shown in Figure 2, eight lines of thermocouples were distributed uniformly throughout the chamber. There were 10 thermocouples in each column, at heights of $0.1 \mathrm{~m}, 0.3 \mathrm{~m}, 0.5 \mathrm{~m}, 0.7 \mathrm{~m}, 0.9 \mathrm{~m}$, 


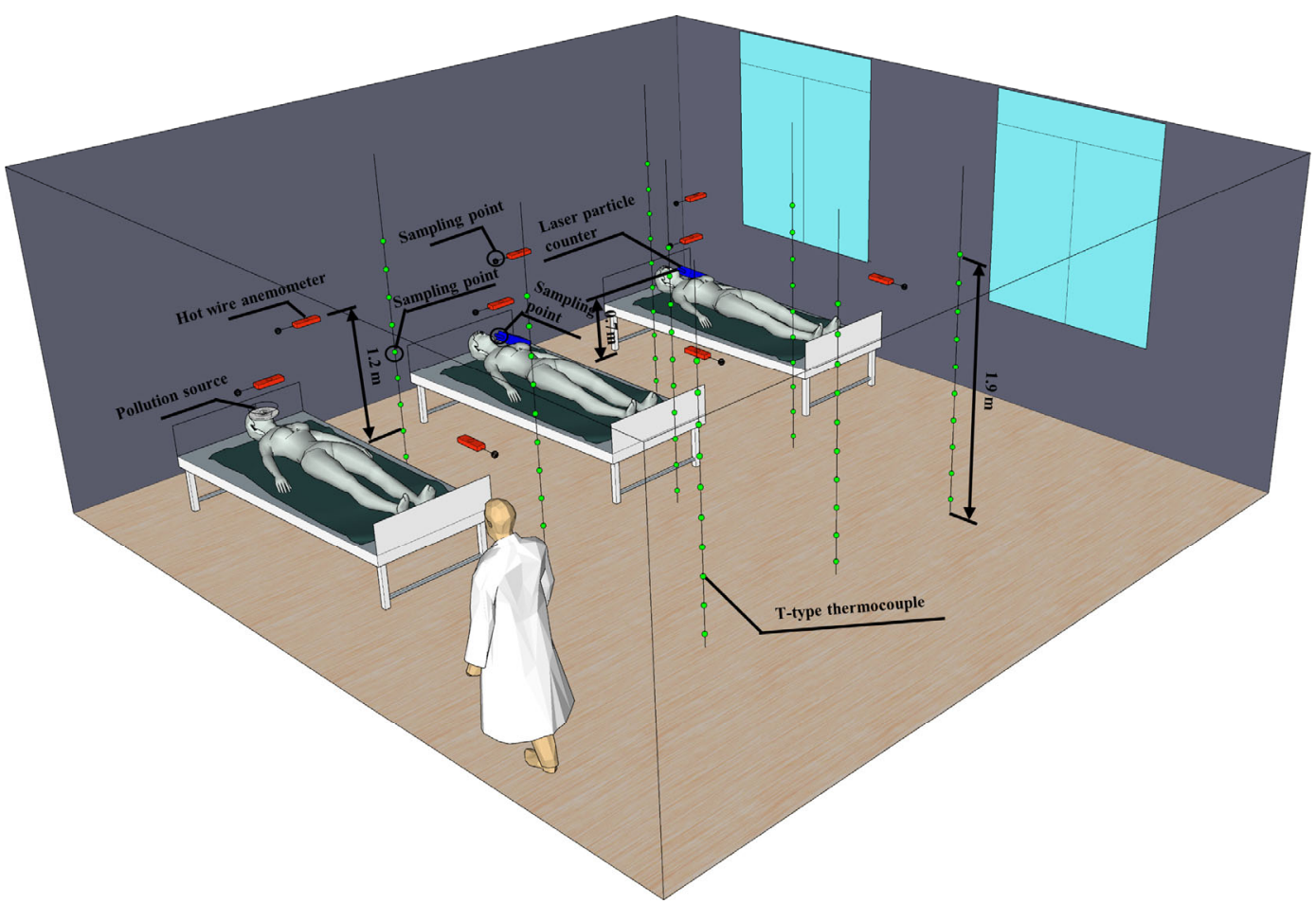

Fig. 2 Locations of instruments used in the experiments

$1.1 \mathrm{~m}, 1.3 \mathrm{~m}, 1.5 \mathrm{~m}, 1.7 \mathrm{~m}$ and $1.9 \mathrm{~m}$, separately. Nine hot-wire anemometers (Model 440, Testo Inc., Lenzkirch, Germany) were used to measure and record the velocity with a frequency of $0.5 \mathrm{~Hz}$. The anemometers were placed $1.2 \mathrm{~m}$ above the ground, along the walking route. The detected wind speed range was $0-5 \mathrm{~m} / \mathrm{s}$ (at a resolution of $0.01 \mathrm{~m} / \mathrm{s}$ ), and the measurement accuracy was $\pm 0.03 \mathrm{~m} / \mathrm{s}$ or $4 \%$ of the measurement value. The particle number concentration was measured by two particle counters (Aerotrak 9306-V2, TSI, Inc., St. Paul, MN, USA) with a frequency of $0.1 \mathrm{~Hz}$, for particles in the size range of $0.3-10 \mu \mathrm{m}$. These two particle counters were placed in the breathing zones of the center and right-hand dummies, $0.7 \mathrm{~m}$ above the ground. The reported particle number concentrations were the average values measured by these two particle counters. All the instruments used in the experiments had been pre-calibrated by the manufacturers. In addition, in order to maintain the consistency of the results, inter-comparisons were conducted between instruments of the same type in pre-test calibrations.

\subsection{Fast Fourier transform (FFT) filtering}

The fast Fourier transform (FFT) was used to process the temperature and air flow field data by means of the following three steps. First, the data was converted into the frequency domain through the FFT; second, the converted data was calculated in the frequency domain using a low- pass filter function (filtering out high-frequency fluctuations and retaining low-frequency effective information); and third, the calculated frequency data was passed through the inverse Fourier transform (IFFT) and converted back to the signal in the time domain. The Fourier transform and its inverse transform are expressed by Eqs. (1) and (2) (Daubechies 1992; Rao et al. 2010):

$$
\begin{aligned}
& x(k)=\sum_{n=0}^{N-1} x(n) W_{N}^{-k n} \\
& x(n)=\sum_{k=0}^{N-1} x(k) W_{N}^{k n}
\end{aligned}
$$

The low-pass filter is defined by Eq. (3):

$w(f)= \begin{cases}1 & f \leq f_{\mathrm{c}} \\ 0 & f>f_{\mathrm{c}}\end{cases}$

where $k, n=0,1, \ldots, N-1 ; W_{N}=\mathrm{e}^{\mathrm{j}(2 \pi / N)} ; \mathrm{j}$ is the imaginary unit; $f_{\mathrm{c}}$ is the cutoff frequency; and $N$ is the number of data points. The FFT filtering process is summarized in Figure 3.

\subsection{Wavelet denoising}

The particle pollutant field data was first discretized by the discrete wavelet transform, and subsequently decomposed and reconstructed based on wavelet multi-resolution analysis (Zhang 2019). The pollutant field data was decomposed into 


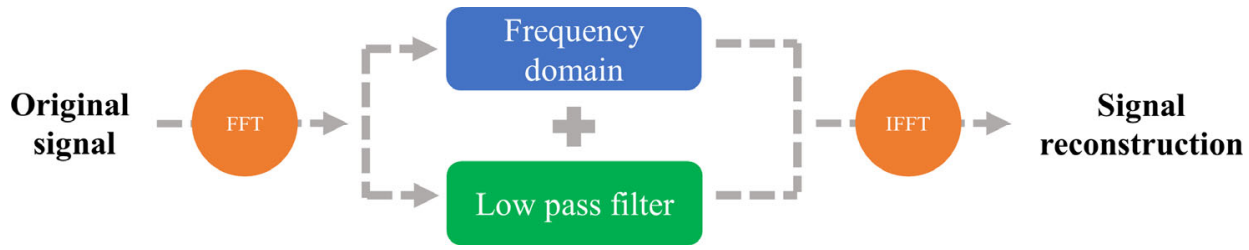

Fig. 3 Flowchart of FFT filtering process

approximate coefficients (low-frequency effective information) and detail coefficients (noise mixed with the low-frequency effective information) by the Mallat algorithm (Resnikoff and Wells 1998). In order to reduce the amount of calculation required for processing discrete data, the pollutant field signal was decomposed into a down-sampling mode and reconstructed into an up-sampling mode, with a scale of $2^{j}$, $j \in \mathbb{Z}$. The approximate coefficients and detail coefficients were calculated by Eqs. (4) and (5) (Ruikar and Doye 2011; Zhao and Cui 2015):

$A_{\mathrm{L}}(n, j)=\sum_{k} W(k, j+1) h(k-2 n)$

$D_{\mathrm{H}}(n, j)=\sum_{k} W(k, j+1) g(k-2 n)$

The wavelet reconstruction process is actually the inverse process of the Mallat algorithm. The reconstruction was carried out by inserting zeros at intervals, as shown in Eq. (6):

$W(n, j+1)=\sum_{k} A_{\mathrm{L}}(k, j) h(n-2 k)+\sum_{k} D_{\mathrm{H}}(k, j) g(n-2 k)$

Here, $A_{\mathrm{L}}$ is the approximate coefficient, $D_{\mathrm{H}}$ is the detail coefficient, $W(k, j+1)=\left\langle X(u), \varphi_{2^{j}}\left(u-2^{-j} k\right)\right\rangle, X(u)$ is the original signal, $\varphi$ is the scale function (Daubechies 1992), and $h(n)$ and $g(n)$ are the low-pass filter coefficients and high-pass filter coefficients, respectively (Daubechies 1992; Kumar and Singh 2015), and $j, n, k \in \mathbb{Z}$. The wavelet denoising process is depicted in Figure 4.

\subsection{Selection of wavelet function}

Unlike the fixed sine function and cosine function of FFT filtering, the basis function of wavelet denoising can be expanded and shifted. At present, there are many kinds of wavelet basis functions from which to choose, and the selection can be based on the following two denoising effect evaluation parameters (Ruikar and Doye 2011; Zhao and Cui 2015; Kumar and Singh 2015; Shemi and Shareena 2016): signal-to-noise ratio (SNR) and mean square error (MSE). These parameters can be calculated by Eqs. (7) and (8).

$$
\begin{aligned}
& \mathrm{SNR}=10 \times \log _{10} \sum_{i=1}^{N} \frac{y_{i}^{2}}{\left(x_{i}-y_{i}\right)^{2}} \\
& \mathrm{MSE}=\frac{1}{N} \sum_{i=1}^{N}\left(y_{i}-x_{i}\right)^{2}
\end{aligned}
$$

In these formulas, $y_{i}$ represents the original signal, and $x_{i}$ represents the processed signal. When a larger SNR and smaller MSE were attained, the wavelet basis function performed better. In addition to the wavelet noise reduction, it was necessary to evaluate the smoothness of the denoising result with the use of Eq. (9) (He et al. 2015). Here, the smaller the $r$ value, the better the degree of smoothing.

$$
r=\frac{\sum_{i=1}^{N-1}[x(i+1)-x(i)]^{2}}{\sum_{i=1}^{N-1}[y(i+1)-y(i)]^{2}}
$$

\subsection{Gaussian function fitting feature extraction}

After filtering and denoising of the experimental data, a smooth curve was obtained. The temperature, velocity and pollutant field fluctuations caused by human walking were fitted by multiple Gaussian functions with high precision. Gaussian fitting is based on either one analytical expression or multiple analytical expressions to approximate discrete data for peak fitting and multi-peak fitting (Guo 2011;

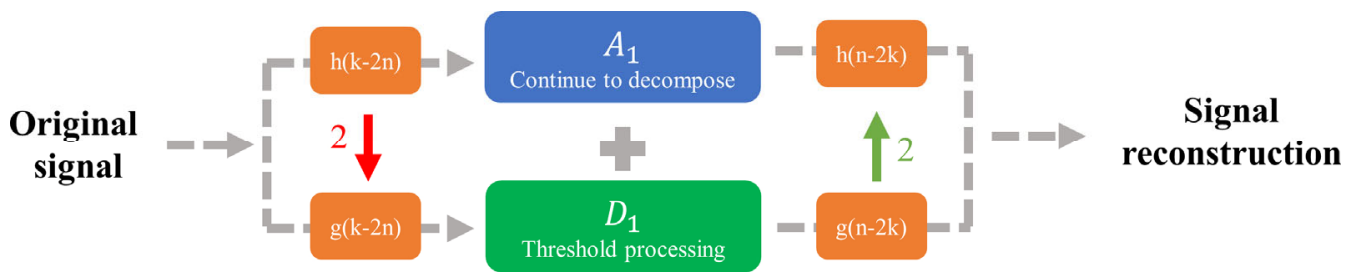

Fig. 4 Flow chart of wavelet denoising process 
Wang et al. 2013). The Gaussian characteristic function is expressed by Eq. (10):

$y_{i}=y_{\max } \mathrm{e}^{-\frac{\left(x_{i}-x_{\max }\right)^{2}}{s}}$

In this formula, $y_{\max }$ represents the peak of the Gaussian curve; $x_{\max }$ represents the position of the peak; and $S$ represents the peak width information. The logarithm of both sides of the above formula was determined and written in the form of a matrix, namely, $Z_{i}=b_{0}+b_{1} x_{i}+b_{2} x_{i}^{2}, Z=$ $\boldsymbol{X B}$. According to the principle of least squares (Wang et al. 2013), the generalized least squares solution of the matrix is $\boldsymbol{B}=\left(\boldsymbol{X}^{\mathrm{T}} \boldsymbol{X}\right)^{-1} \boldsymbol{X}^{\mathrm{T}} \boldsymbol{Z}$. Solving the coefficient matrix $\boldsymbol{B}$ allowed the Gaussian characteristic function to be solved (Guo 2011). Thus, the eigenvalues $S_{i}$ and $y_{\max , i}$ that characterize the disturbance time and the disturbance range were obtained. The eigenvalues of the dimensionless particle concentration scale and the eigenvalues of the dimensionless time scale were then calculated and used to quantitatively analyze the robustness of the ventilation system. The FFT filtering, wavelet denoising and Gaussian function fitting in this study were conducted by Origin 2018 (OriginLab, Northampton, MA, USA).

\subsection{Analysis of ventilation system robustness in response to range and time scale fluctuations}

The range scale eigenvalue $\left(y_{\max , i}\right)$ represents the disturbance to the indoor field caused by people's walking, and the time scale eigenvalue $\left(S_{i}\right)$ represents the time required for the indoor field to return to its original state when the walking has stopped. Once the disturbance time and range eigenvalues under different ventilation systems were obtained, the robustness of the ventilation systems in response to walking-induced disturbance fluctuations could be determined. The robustness represents the control effectiveness of a ventilation system on the range and time scales. For easier comparison of the robustness of different ventilation systems, the disturbance time and range eigenvalues were dimensionlessly processed and transformed into index evaluation values. At the same time, for a more intuitive analysis of the robustness of the system, a linear dimensionless method was used in this study (Guo 2011). The specific gravity method was used in the dimensionless method to construct robustness indicators on the range and time scales. The evaluation index represents a linear relationship. When exploring a system's robustness in controlling the pollutant field disturbance, it was necessary to consider the average particle concentration when the system was stable. The analysis of the robustness of ventilation systems in response to temperature and flow field range scale fluctuations is expressed by Eq. (11), and for the pollutant field it is expressed by Eq. (12). The robustness for all three fields in response to time scale fluctuations is uniformly expressed by Eq. (13).

$$
\begin{aligned}
D_{\mathrm{R}, 1}= & 1-\left[\frac{\operatorname{Avg}_{k}\left(y_{\text {max }, 1}, y_{\max , 2}, \ldots, y_{\max , n}\right)}{\sum_{k=1}^{n} \operatorname{Avg}_{k}\left(y_{\max , 1}, y_{\text {max }, 2}, \ldots, y_{\max , n}\right)}\right] \\
D_{\mathrm{R}, 2}= & 1-\left[\frac{\operatorname{Avg}_{k}\left(y_{\text {max }, 1}, y_{\text {max }, 2}, \ldots, y_{\text {max }, n}\right)}{2 \sum_{k=1}^{n} \operatorname{Avg}_{k}\left(y_{\text {max }, 1}, y_{\text {max }, 2}, \ldots, y_{\max , n}\right)}\right. \\
& \left.+\frac{\operatorname{Avg}_{k}\left(P_{1}, P_{2}, \ldots, P_{n}\right)}{2 \sum_{k=1}^{n} \operatorname{Avg}_{k}\left(P_{1}, P_{2}, \ldots, P_{n}\right)}\right] \\
D_{\mathrm{T}}= & 1-\frac{\operatorname{Avg}_{k}\left(S_{1}, S_{2}, \ldots, S_{n}\right)}{\sum_{k=1}^{n} \operatorname{Avg}_{k}\left(S_{1}, S_{2}, \ldots, S_{n}\right)}
\end{aligned}
$$

where $P$ is the baseline of Gaussian fitting, which represents the effectiveness of the ventilation system in removing particulate matter (the calculated range scale value is above this baseline), and $\mathrm{Avg}_{k}$ is the average value of range scale $y_{\max , i}$ and time scale $S_{i}$ under different system conditions.

The range scale for $D_{\mathrm{R}, 1}, D_{\mathrm{R}, 2}$, and $D_{\mathrm{T}}$ was from 0 to 1 . The larger the robustness value, the more robust the system, and the better the system could deal with the disturbance caused by human walking. Based on the system robustness evaluation index for the range scale and time scale, it was feasible to explore the impact of human movements on the four different types of ventilation systems under the same environmental conditions. The algorithm and diagram for data noise reduction, interference feature extraction and robustness calculation are summarized in Figure 5.

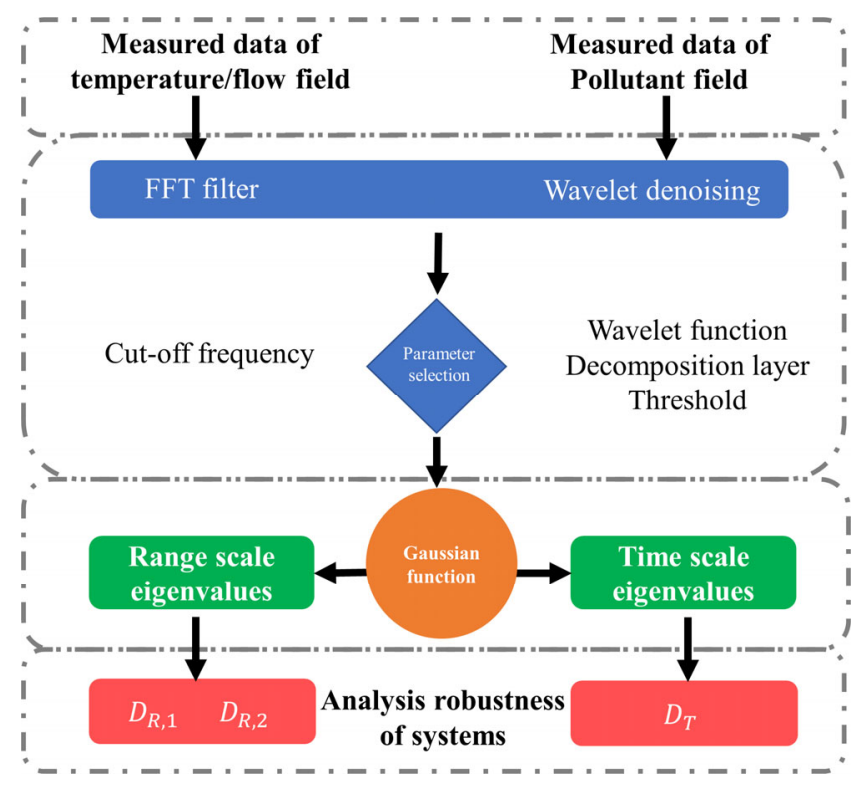

Fig. 5 The flow chart for the overall data processing 


\section{Results}

\subsection{Noise reduction in temperature and flow fields}

Figure 6 presents one example of the field data measured under the ceiling supply and ceiling return ventilation system. In this study, we focused on the selection of noise reduction and feature extraction methods for the fluctuation. FFT was used to convert the temperature and flow field signals shown in Figures 6(a) and (b) to the frequency domain spectrum shown in Figure 7 . In the latter figure, the distribution of low-frequency high-amplitude signals and high-frequency low-amplitude signals can be clearly observed. The high-frequency low-amplitude signals were noise and needed to be filtered. The low-frequency and high-amplitude signals contained useful information about temperature and flow field fluctuation caused by human walking, and needed to be extracted (He et al. 2015).

It can be seen in Figures 6 and 7 that the existence of a large amount of high-frequency noise would directly affect the results of the quantitative analysis of flow disturbance. A good filter effect can be achieved by selecting an appropriate cut-off frequency in the high-amplitude region. With the walking frequency as a reference, the cut-off frequency for the temperature field was set as $0.004 \mathrm{~Hz}$, and that for the flow field was set as $0.005 \mathrm{~Hz}$. The temperature and flow fields after noise reduction are shown in Figure 8. The selection of cut-off frequency is very important for the
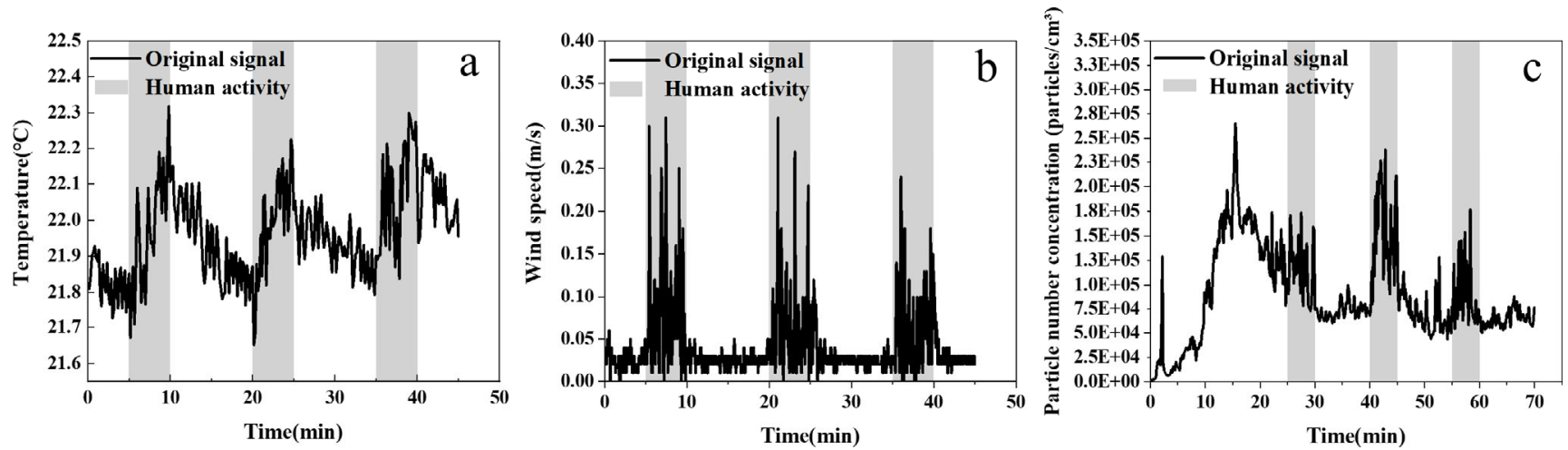

Fig. 6 The measured (a) temperature, (b) flow and (c) pollutant fields under ceiling supply and ceiling return ventilation systems
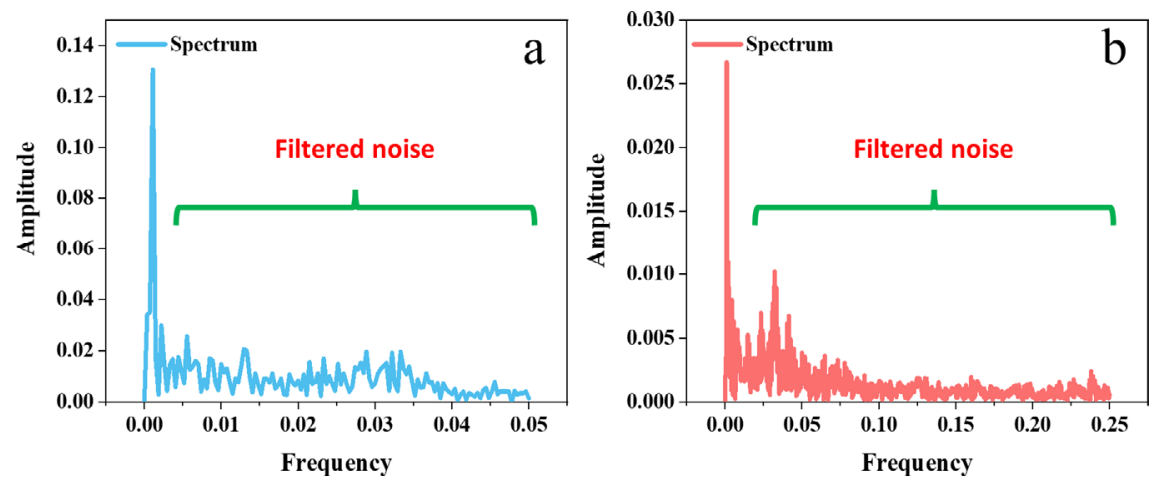

Fig. 7 Spectrums of (a) temperature and (b) flow field signal
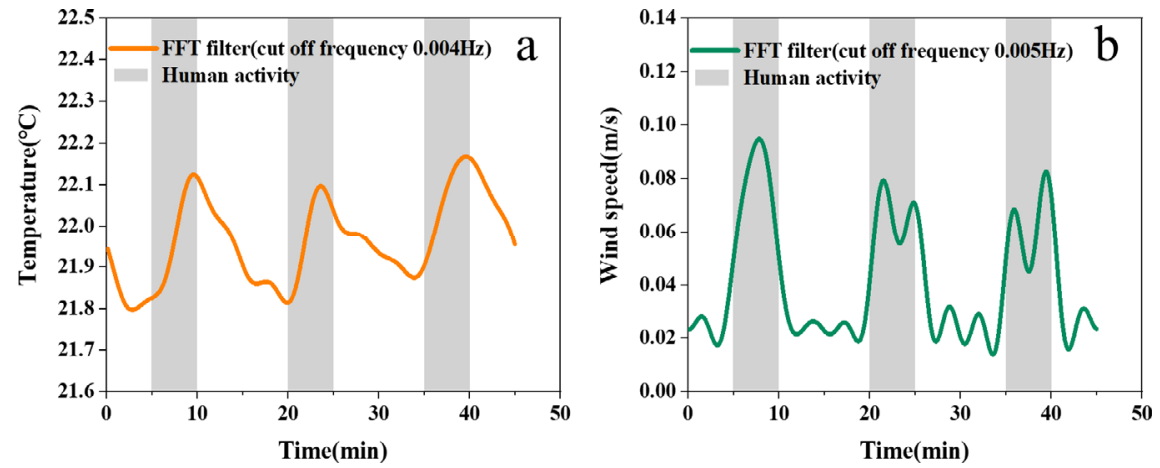

Fig. 8 (a) Temperature and (b) flow field after noise reduction 
denoising effect, and will be further discussed in the following section.

\subsection{Noise reduction in pollutant field}

Wavelet denoising was used to process the non-periodic pollutant field as shown in Figure 6(c). As described above, the wavelet basis function is fundamental to the wavelet denoising process. In this study, the DB5 wavelet function (Ngui et al. 2013) was selected as the basis function. The reason for this selection will be provided in the following section. In accordance with the low-pass filter coefficient $h(n)$ and high-pass filter coefficient $g(n)$ obtained by the Daubechies wavelet, the pollutant field signal was decomposed into an approximate coefficient and detail coefficient based on the Mallat algorithm (Resnikoff and Wells 1998). The approximate coefficient corresponds to large-scale and lowfrequency pollutant field signals, and the detail coefficient corresponds to small-scale and high-frequency pollutant field signals. The threshold was applied to the detail coefficient to reconstruct the pollutant field signal.

By means of the wavelet transform, the original data was down-sampled and decomposed. Each sampling divided the pollutant field signal into a low-frequency part and a relatively high-frequency part. In this study, the pollutant field signal was decomposed four times to obtain a set of approximate coefficients and group detail coefficients, as shown in Figure 9. Next, based on the decomposed detail coefficients and a custom threshold value of $55.5 \%$ for each level, the detail coefficients with low coefficient values were filtered.

The wavelet reconstruction process is the inverse process of the Mallat algorithm (Resnikoff and Wells 1998). Therefore, the wavelet reconstruction is an up-sampling process, and the original signal is reconstructed by interpolation of zero-value points, as shown in Figure 10. A comparison of the pollutant signals in Figure 6(c) and Figure 10 indicates that the pollutant field that was reconstructed by wavelet de-noising retained its local characteristics with good noise-reduction effect.

\subsection{Disturbance feature extraction}

After the above FFT and wavelet denoising, smooth curves for temperature, flow and pollutant field signals were attained, as shown in Figure 11. The curves were fitted by a Gaussian function (Guo 2011; Wang et al. 2013), and the analytical expressions were subsequently obtained. The analytical formula fitted by the Gaussian function was then used to calculate the disturbance time $S_{i}$ and the disturbance
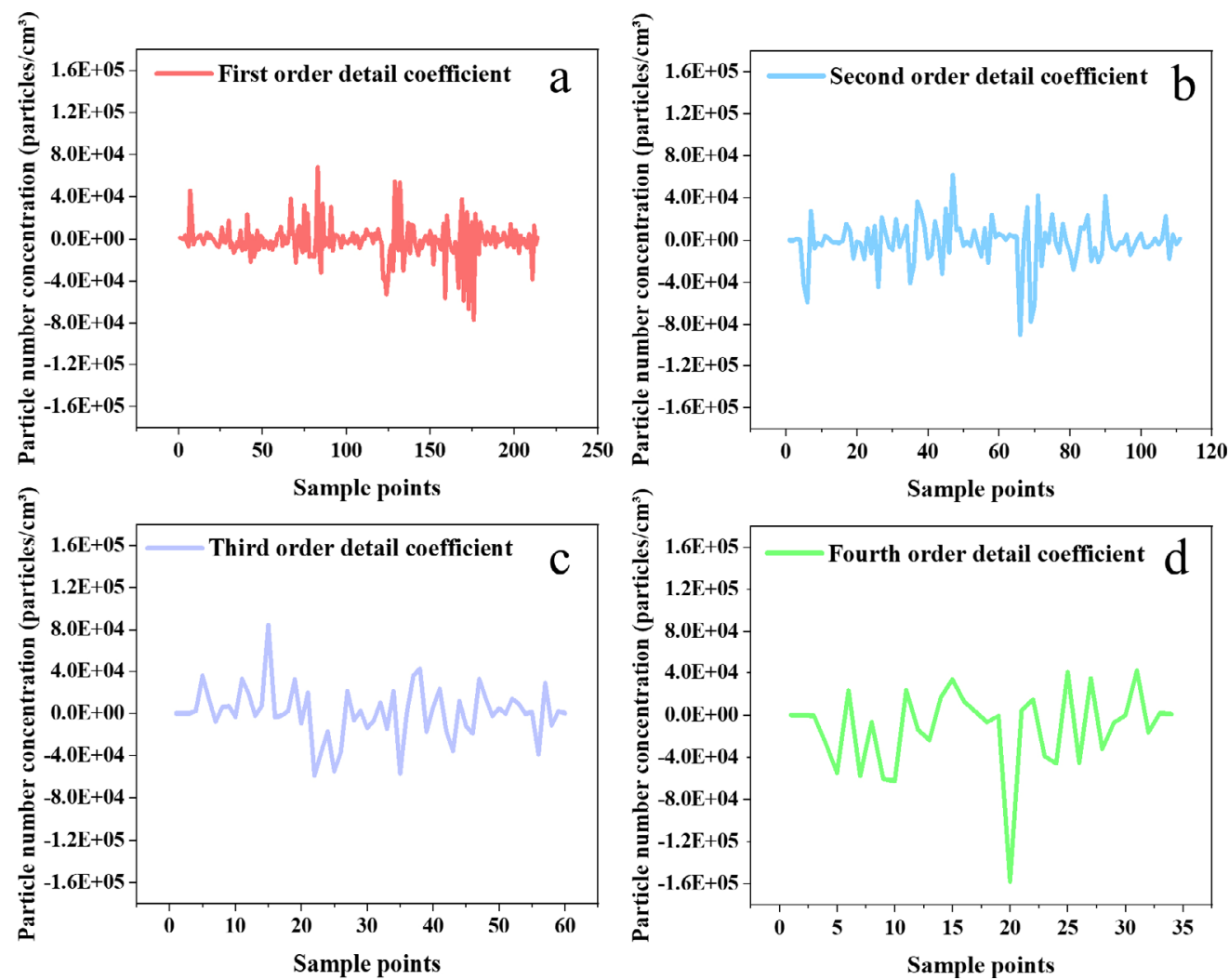

Fig. 9 (a) First-order detail coefficient, (b) second-order detail coefficient, (c) third-order detail coefficient, and (d) fourth-order detail coefficient for wavelet denoising 


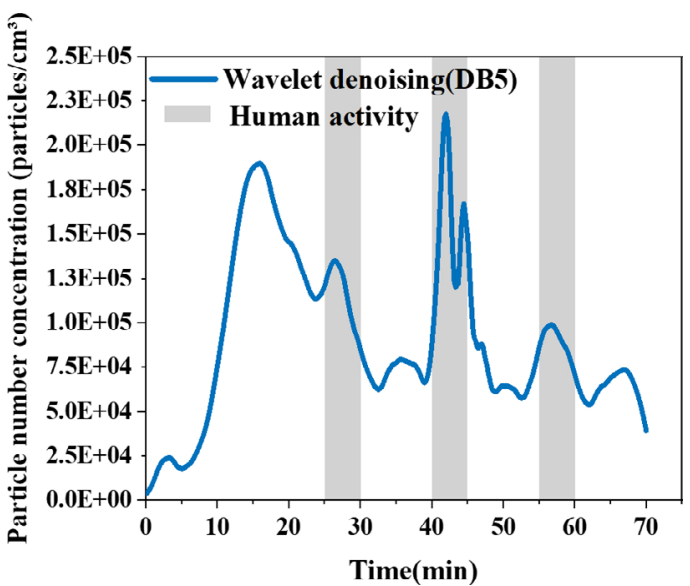

Fig. 10 Pollutant field signal after noise reduction

range $y_{\max , i .}$ The corresponding characteristic values for field signals in Figure 11 were calculated and are provided in Table 1.

\subsection{Analysis of ventilation system robustness}

Figure 12 displays the Gaussian fitting curves for the temperature, flow and pollution field averages in the breathing zones of the center and right-hand dummies under different ventilation systems. Even though the air exchange rate of the ventilation system was identical, and the human walking parameters consistent, under the different systems, significant differences were observed in the walking-induced fluctuations in the temperature, field and pollutant fields. The range scale and time scale robustness were calculated by Eqs. (11)-(13) and are summarized in Table 2.

For the temperature field, the range scale and time scale robustness values for CS were the smallest, while similar values were found for CC, SC and SS. There are two possible reasons for this: first, multiple ventilation systems performed well in indoor temperature control; and second, one person's heat loss was relatively small, and the human walking had limited effect on the overall indoor temperature field. For the flow field, when the ventilation system was not operating, the disturbance caused by walking was much larger than it was when the system was operating, as depicted in Figure 12. Furthermore, as shown in Table 2, the use of the ventilation system could increase the range scale robustness value by $19.7 \%-39.4 \%$ compared with the value when the ventilation system was off. The largest range scale robustness value was achieved by the SS system, and it was $16.5 \%$ higher than the
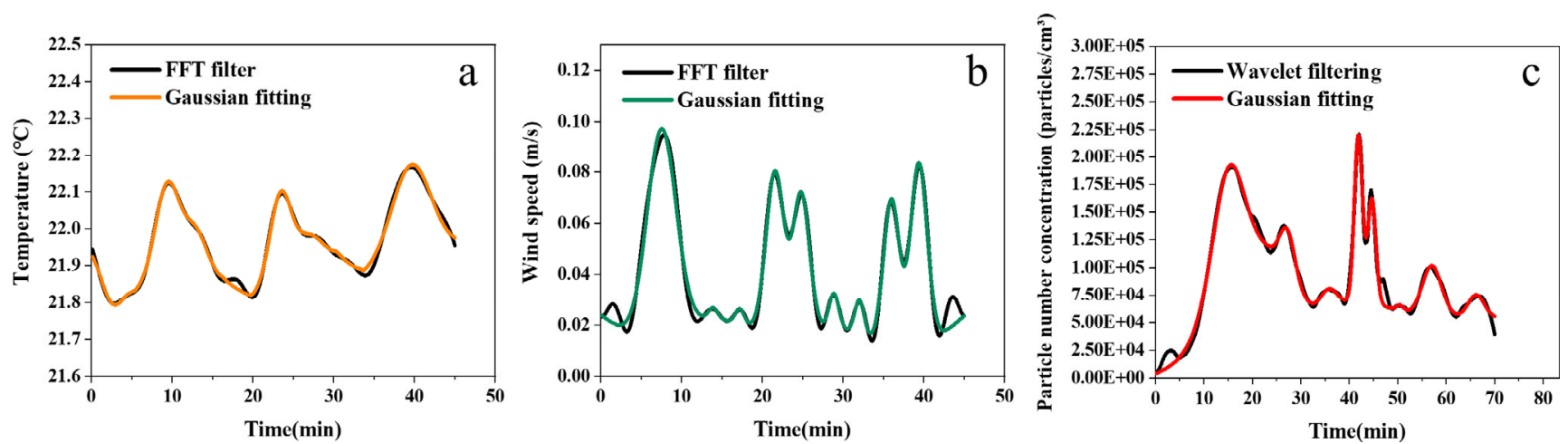

Fig. 11 Gaussian fitting for (a) temperature, (b) flow and (c) pollutant fields

Table 1 Summary of the range and time characteristic values

\begin{tabular}{|c|c|c|c|}
\hline \multirow[b]{2}{*}{ Field type } & \multirow[b]{2}{*}{ Walking scenario } & \multicolumn{2}{|c|}{$e^{-\frac{\left(x_{i}-x_{\max }\right)^{2}}{2 S_{i}}}$} \\
\hline & & Range scale, $y_{\max , i}$ & Time scale, $2 S_{i}$ \\
\hline \multirow{3}{*}{ Temperature } & $1^{\text {st }}$ walk & $0.31{ }^{\circ} \mathrm{C}$ & $14.2 \mathrm{~min}$ \\
\hline & $2^{\text {nd }}$ walk & $0.35^{\circ} \mathrm{C}$ & $14.2 \mathrm{~min}$ \\
\hline & $3^{\text {rd }}$ walk & $0.34{ }^{\circ} \mathrm{C}$ & $11.4 \mathrm{~min}$ \\
\hline \multirow{3}{*}{ Flow } & $1^{\text {st }}$ walk & $0.10 \mathrm{~m} / \mathrm{s}$ & $8.4 \mathrm{~min}$ \\
\hline & $2^{\text {nd }}$ walk & $0.09 \mathrm{~m} / \mathrm{s}$ & $10.0 \mathrm{~min}$ \\
\hline & $3^{\text {rd }}$ walk & $0.09 \mathrm{~m} / \mathrm{s}$ & $9.5 \mathrm{~min}$ \\
\hline \multirow{3}{*}{ Pollutant } & $1^{\text {st }}$ walk & $9.30 \mathrm{E}+04$ particles $/ \mathrm{cm}^{3}$ & $7.3 \mathrm{~min}$ \\
\hline & $2^{\text {nd }}$ walk & $1.01 \mathrm{E}+05$ particles $/ \mathrm{cm}^{3}$ & $7.9 \mathrm{~min}$ \\
\hline & $3^{\text {rd }}$ walk & $9.46 \mathrm{E}+04$ particles $/ \mathrm{cm}^{3}$ & $9.3 \mathrm{~min}$ \\
\hline
\end{tabular}



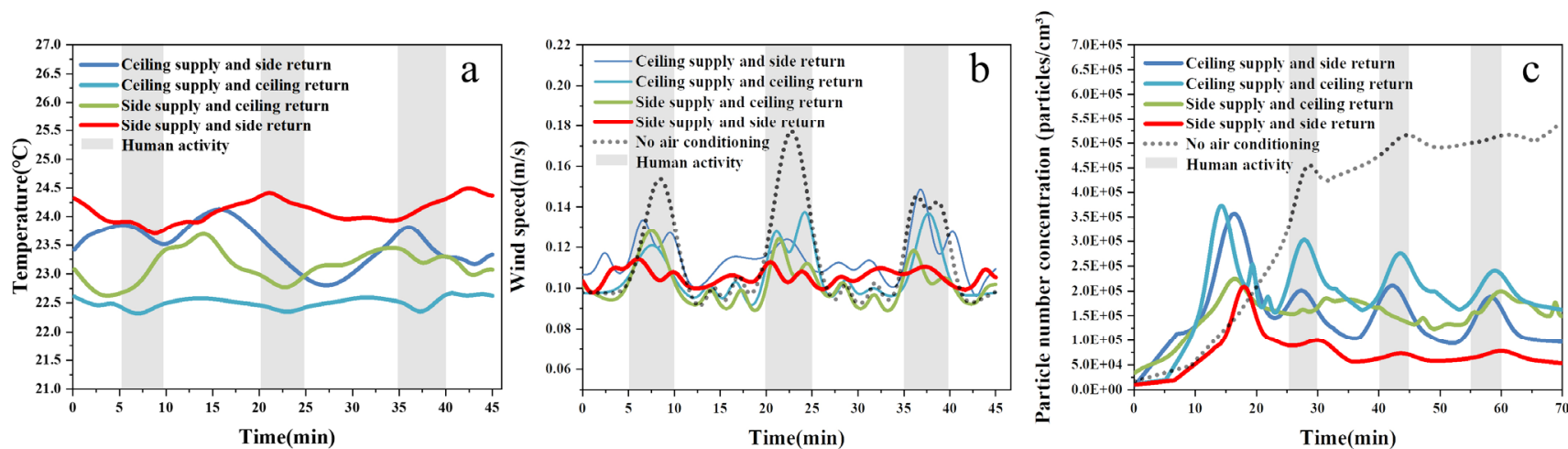

Fig. 12 Gaussian fitting curves for (a) temperature, (b) flow and (c) pollutant fields under different ventilation systems

Table 2 Range scale and time scale robustness under different ventilation systems

\begin{tabular}{clcc}
\hline & & Range scale robustness, $D_{\mathrm{R}}$ & Time scale robustness, $D_{\mathrm{T}}$ \\
\hline \multirow{3}{*}{ Temperature field } & Ceiling supply, side return & 0.60 & 0.61 \\
& Ceiling supply, ceiling return & 0.87 & 0.79 \\
& Side supply, ceiling return & 0.70 & 0.79 \\
& Side supply, side return & 0.77 & 0.80 \\
\hline \multirow{2}{*}{ Flow field } & Ceiling supply, side return & 0.81 & 0.81 \\
& Ceiling supply, ceiling return & 0.79 & 0.81 \\
& Side supply, ceiling return & 0.81 & 0.79 \\
& Side supply, side return & $\mathbf{0 . 9 2}$ & 0.80 \\
& No ventilation system & 0.66 & 0.79 \\
\hline \multirow{2}{*}{ Pollution field } & Ceiling supply, side return & 0.79 & 0.78 \\
& Ceiling supply, ceiling return & 0.74 & 0.81 \\
& Side supply, ceiling return & 0.86 & 0.84 \\
& Side supply, side return & $\mathbf{0 . 9 3}$ & 0.79 \\
\end{tabular}

smallest value. However, the time scale robustness values under different conditions were similar. Thus, the ventilation system performed well in controlling the disturbance range, but might have a very limited effect in controlling the disturbance time. For the pollutant field, when the ventilation system was off, the particle concentration in the chamber increased consistently, possibly due to the operation of the particle generator. The particle concentrations under different ventilation systems were much lower than those observed in the absence of ventilation. The use of the ventilation system increased the range scale robustness value by $10.0 \%-38.8 \%$ compared with the value when the ventilation system was off. The largest range scale robustness value was also achieved by the SS system, and it was $25.7 \%$ higher than the lowest value. As with the temperature field, the ventilation system had a very limited effect in controlling the disturbance time for the pollutant field. Kong et al. (2021) studied the effectiveness of different ventilation systems in the control of fine particles in the same chamber. They found that a side supply and side return ventilation system performed the best in controlling personal exposure to particles. The present study demonstrated that this type of ventilation system not only reduces particle exposure but can also control the concentration fluctuation, which has a significant impact on indoor air quality control.

Besides, the flow field of the SS system achieved high robustness, while the pollutant field also achieved high robustness. It was not surprising to find these results. Many studies indicated that flow field play an important role to reduce the diffusion of particles (Liu et al. 2020; Lv et al. 2021; Massarotti et al. 2021). However, the robustness of the flow field could not completely determine the distribution of particles. Such as the CS, CC and SC systems, their robustness (0.79-0.81) of the flow field were similar, but their robustness of the pollutant field have certain difference (0.74-0.86). One possible reason was the different particle filtration efficiency between the ventilation systems (Agirman et al. 2020; Kong et al. 2021). Therefore, according to our 
sampling results, particle filtration efficiency and stable flow field were essential conditions for high robustness of ventilation systems, such as SS system.

\section{Discussion}

\subsection{Analysis of filtering feasibility}

If there were stable or non-stationary and irregular highfrequency fluctuations in the measured data, the analysis of effective signal components would be strongly affected. The principle of FFT and wavelet analysis is to transform the signal into the frequency domain for analysis, and modify the signal components as the superposition of a series of basis functions (Daubechies 1992).

The experiments in this study demonstrated that FFT filtering and wavelet noise reduction can extract signal features of different frequencies reliably and accurately according to the characteristics of sampling frequency and data fluctuations. Different filters can be used to extract different signal features. For instance, a low-pass filter for FFT was applied in this study to extract low-frequency signals. In addition to the low-pass filter, there are also a high-pass filter (to extract high-frequency signals) and a band-pass filter (a filter that can set the lower and upper limits of the frequency). According to the needs of a given situation, different filters can be used to analyze the experimental data. For feature extraction, wavelet noise reduction is equipped with both a low-pass filter and a high-pass filter for multi-resolution analysis. Therefore, according to the sampling frequency characteristics of the effective information, different filters can effectively extract different signal characteristics.

\subsection{Selection of FFT filtering or wavelet transform}

The wavelet transform is an analysis method that is based on the Fourier transform, and it takes optimization into consideration. According to results obtained by Sifuzzaman et al. (2009) the wavelet transform performs better than the Fourier transform. The wavelet transform is an existing detail-based method with a shorter response time, making it more suitable for high-precision online verification (Sifuzzaman et al. 2009; Gothwal et al. 2011). The basis function used by FFT filtering is an infinite loop, and there is no area limitation, whereas the wavelet function used by wavelet noise reduction is limited. Therefore, FFT filtering is overall filtering, while wavelet noise reduction can capture the time domain information. As shown in the frequency spectrum of Figure 7, when the cutoff frequency is determined, the low-pass filter of FFT filters all parts higher than the cutoff frequency, which inevitably leads to signal distortion. The wavelet basis function is defined in a limited interval, and multi-scale analysis can be performed at the same time. Therefore, while filtering the noise below the cut-off frequency, it retains some essential details and increases the accuracy of the filtering.

As shown in Figure 13, FFT filtering and wavelet denoising were used to denoise the pollutant field with no obvious periodicity or local mutations. According to the Nyquist sampling law (Daubechies 1992; Liu and Han 2018), the number of decomposition layers of wavelet denoising is 4 , and the cutoff frequency of FFT filtering is between $0.003 \mathrm{~Hz}$ and $0.0048 \mathrm{~Hz}$. When the cutoff frequency is $0.004 \mathrm{~Hz}$, the pollutant field will be distorted. When the cutoff frequency is increased to $0.008 \mathrm{~Hz}$, not only is the accuracy not as high as with wavelet noise reduction, but excess noise is also observed. This is because the use of FFT in converting the signal to the frequency domain is not based on information in the time domain, and cannot accurately locate and reduce the noise of the local mutations. Therefore, the accuracy of the reconstruction is also affected. When the wavelet multi-discrimination analysis is used to denoise the pollutant field signal, the wavelet function can
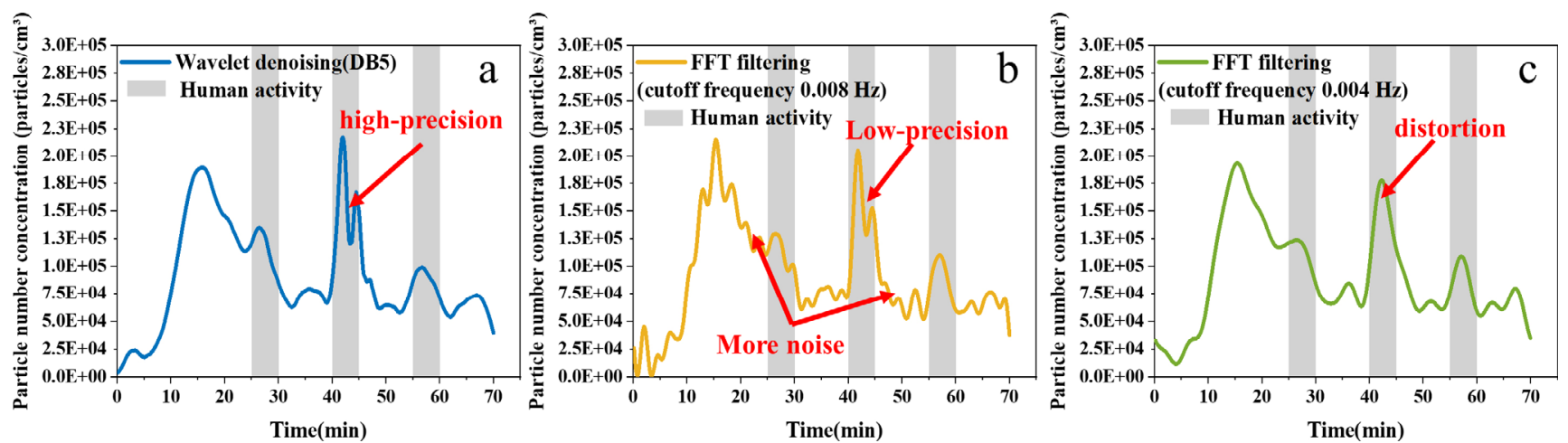

Fig. 13 Pollutant field after noise reduction: (a) DB5, 4-layer decomposition, custom threshold: 55.5\%; (b) FFT, cut off frequency: 0.008 Hz; (c) FFT, cut off frequency: $0.004 \mathrm{~Hz}$ 
be stretched and shifted, and therefore the local mutation information is captured and the high-precision reconstruction is carried out with less noise.

As shown in Figure 14, for the temperature field, although some information is lost with the use of the FFT filter, the range scale feature quantity and the time scale feature quality are extracted with very limited effect. The approximate periodic temperature field and flow field also conform to the periodic nature of the FFT filtering basis functions, the sine and cosine functions. Therefore, the cutoff frequency can be set directly for FFT filtering. When the wavelet function is used to process the signal, then the wavelet function, the number of decomposition layers and the threshold are not selected at once, and the complexity of the signal must be adjusted. The calculation process for the wavelet function was found to be more cumbersome. This study needed to further extract the characteristic quantities on the time scale and concentration range scale. Therefore, when processing the pollutant field signal, it is necessary to use wavelet analysis for high-precision reconstruction to accurately calculate the exposure. For the temperature and flow fields, it is necessary only to extract the feature quantities on the time scale and the range scale. Therefore, in this study, the temperature field and flow field were filtered by FFT, and the pollutant field was processed by wavelet noise reduction.

\subsection{Selection of FFT filter cut-off frequency}

The cut-off frequency of the FFT filter needs to meet the Nyquist sampling law (Daubechies 1992; Liu and Han 2018); that is, the sampling frequency of the signal must be more than twice the highest frequency. The relationship between the sampling frequency and the highest frequency of the signal characteristics is shown in Figure 15. In order to fully recover the low-frequency signal of people's walking, at least 7 sampling points are required, and the highest frequency should not be less than $0.0015 \mathrm{~Hz}$. In fact, in order to retain more details to improve the accuracy of signal reconstruction, the frequency of actual sampling is generally 2.56-4 times the highest frequency ( $\mathrm{Wu}$ and Hua 2014). To obtain more details, the cutoff frequency should be higher than 2.56-4 times the highest frequency of the characteristic signal of people's walking. In Figures 15(b) and (c), the denoising effect and accuracy for two different cut-off frequencies in this range are compared. When the cut-off frequency is lower than or equal to $0.001 \mathrm{~Hz}$, serious distortion will occur. In comparison, if the cut-off frequency is higher than or equal to $0.005 \mathrm{~Hz}$, noise will start to appear. Therefore, the cut-off frequency should be higher than $0.001 \mathrm{~Hz}$ and lower than $0.005 \mathrm{~Hz}$. In this study, the temperature field was denoised by the FFT filter with a cut-off frequency of $0.004 \mathrm{~Hz}$, and similarly, the cut-off
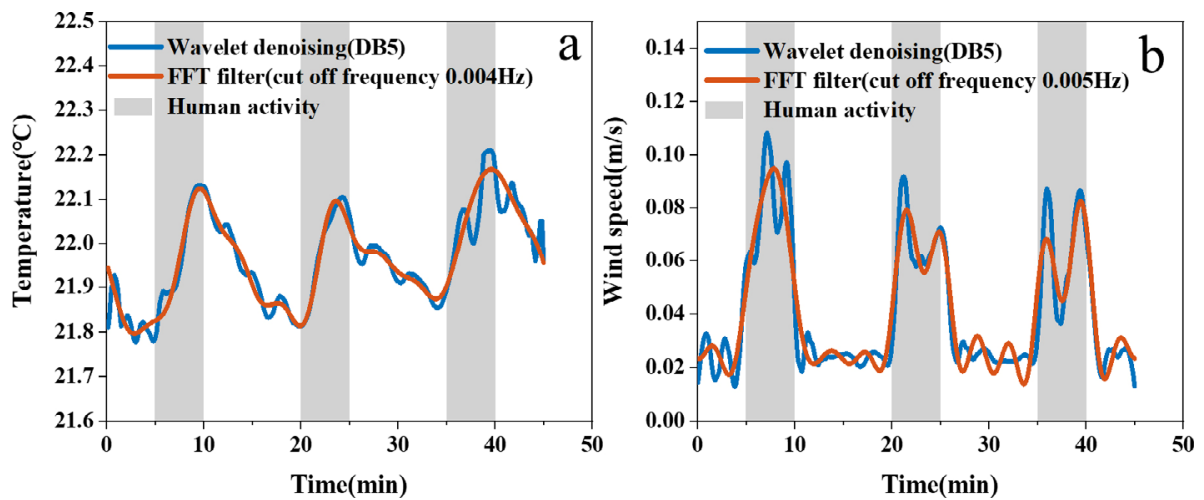

Fig. 14 Comparison of FFT filtering and wavelet denoising for (a) temperature field and (b) flow field
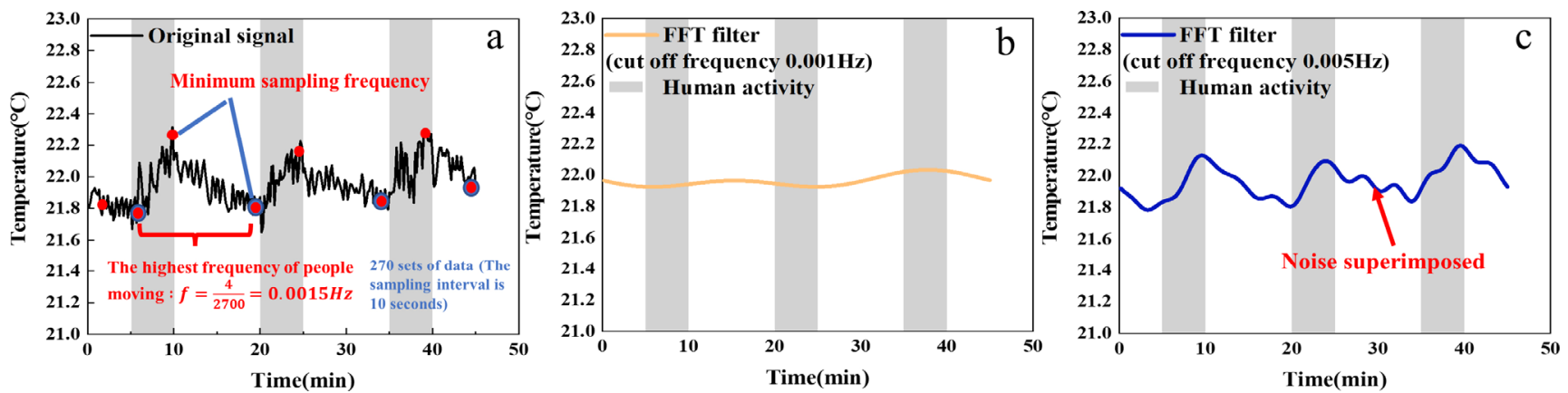

Fig. 15 (a) The relationship between the largest frequency of the temperature field and the sampling frequency; (b) temperature field after noise reduction (FFT, cut-off frequency: $0.001 \mathrm{~Hz}$ ); (c) temperature field after noise reduction (FFT, cut-off frequency: $0.005 \mathrm{~Hz}$ ) 
frequency for the flow field was set as $0.005 \mathrm{~Hz}$. This difference was caused by the fact that the sampling frequency for the flow field was higher than for the temperature field. The cut-off frequency for FFT denoising can be adjusted according to the above method if a different human walking frequency or sampling frequency was reported in other studies.

\subsection{Selection of wavelet function}

The selection of the wavelet function is related to the shape of the original signal. The more similar the shape of the wavelet function is to the original, the better the accuracy (Ruikar and Doye 2011). The selection of wavelet function can evaluate the denoising effect basing on the calculated values of SNR and MSE. A larger SNR and smaller MSE indicate better denoising effects (Sifuzzaman et al. 2009; Gothwal et al. 2011; Guo 2011; Shemi and Shareena 2016). Because the noise reduction effect is influenced by the number of decomposition layers and thresholds, 4 levels of decomposition were used in this study, and the custom threshold was set at $100 \%$. The optimal wavelet function was then selected on the basis of the calculated SNR and MSE values, as shown in Table 3. Once the wavelet function was determined, the number of decomposition levels and the threshold could be selected.

According to Table 3, the DB2 wavelet could be selected to denoise the pollutant field, due to its large SNR and small MSE. However, the smoothness and accuracy of the resulting curve are not as good as those obtained by DB5 wavelet denoising, as shown in Figure 16.

Therefore, the use of SNR and MSE in evaluating the noise reduction effect is not all-encompassing. It is true that the smaller the MSE value, the closer the signal after

Table 3 SNR, MSE and $r$ values for different wavelet functions

\begin{tabular}{cccc}
\hline Wavelet function & SNR & MSE & $r$ \\
\hline DB2 & 93.6 & 2037 & 22294 \\
DB3 & 67.3 & 153242 & 23964 \\
DB4 & 74.4 & 63176 & 42098 \\
DB5 & 77.5 & 7153 & 13894 \\
DB6 & 69.8 & 237467 & 19040 \\
DB7 & 71.4 & 44367 & 15890 \\
DB8 & 80.4 & 35211 & 10075 \\
DB9 & 73.3 & 243030 & 21013 \\
DB10 & 58.3 & 31460 & 13769 \\
Bior3.1 & 65.7 & 170187 & 39139 \\
Bior3.3 & 66.2 & 116973 & 9942 \\
Bior3.5 & 66.2 & 43 & 15026 \\
Bior3.7 & 67.1 & 580 & 14884 \\
\hline
\end{tabular}

noise reduction is to the original signal. However, if the signal is not denoised, or if the residual noise in the signal is closer to the original signal, the value of MSE will also be small. Therefore, the smoothness of the denoising result should also be taken into consideration (He et al. 2015). The selection of wavelet function is comprehensively considered by integrating SNR, MSE and smoothness $r$, as shown in Table 3. Compared with other wavelet functions, the DB5 wavelet function (Deng and Jiang 2012) has better comprehensive performance and high fitting accuracy.

\subsection{Selection of decomposition layers and threshold for wavelet denoising}

The wavelet denoising process decomposes the samples step by step with a scale of $2^{j}$. For each decomposing time, the number of signal samples is halved until the signal samples are exhausted. At the same time, a certain threshold is applied to each level after decomposition, so that noise reduction can be performed at different frequency levels. Theoretically, the number of wavelet decomposition layers is related to the number of original data sample points (Mallat 1989). The greater the number of decomposition layers, the more noise is filtered out. If there are too many decomposition layers, signal distortion will result. Similarly, according to the Nyquist sampling law, as shown in Figure 17(a), when the wavelet noise reduction decomposes the signal, the low-frequency fluctuations caused by the movement of people must be completely preserved. At least 9 sampling points are required. The highest frequency of the feature should not be smaller than $0.0012 \mathrm{~Hz}$. The appropriate frequency should be a multiple of the highest frequency by $2.56-4$ times, i.e., approximately in the range of $0.003-0.0048 \mathrm{~Hz}$. Each decomposition will divide the signal frequency into a high-frequency part and a lowfrequency part. Since the total frequency of the pollutant field is $0.1 \mathrm{~Hz}$, when the four-layer decomposition is used, the total frequency of the signal is decomposed to $0-0.0063 \mathrm{~Hz}$, which conforms to Nyquist Stuart's sampling law (Daubechies 1992; Liu and Han 2018).

The selection of thresholds also affects the wavelet denoising results. Currently, three types of thresholds are widely used: a hard threshold, a soft threshold and a custom threshold (Sifuzzaman et al. 2009; Shemi and Shareena 2016). All of these threshold types have advantages and disadvantages. The signal after soft-threshold processing will not produce additional oscillation, and the smoothness is better than with the hard threshold. In comparison, the signal after the hard-threshold processing is closer to the original signal, and it is better than the soft-threshold method in the sense of smaller mean square error. However, once the hard threshold or soft threshold is selected, there 

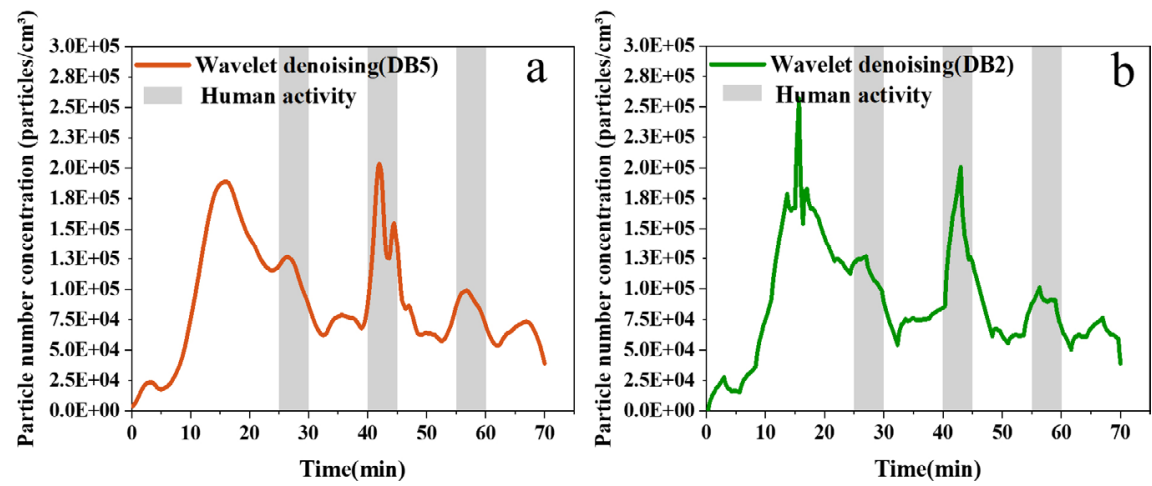

Fig. 16 Pollutant field after noise reduction (a) DB5, 4-layer decomposition, custom threshold: 100\%; (b) DB2, 4-layer decomposition, custom threshold: $100 \%$
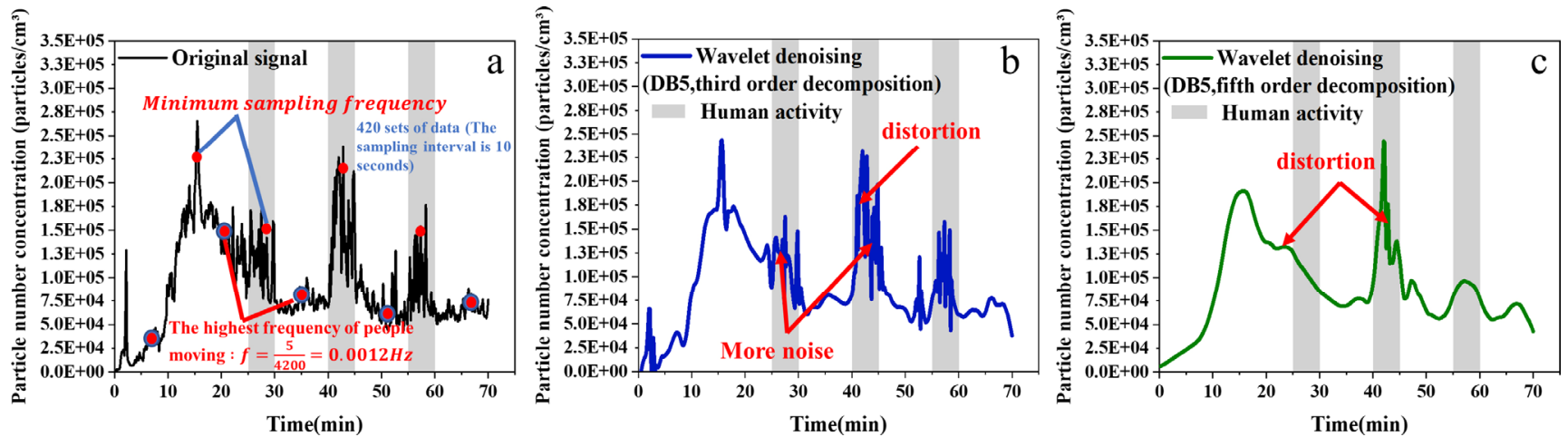

Fig. 17 (a) The relationship between the highest frequency of the pollutant field and the sampling frequency; (b) pollutant field after noise reduction (DB5, 3-layer decomposition, custom threshold: 55.5\%); (c) pollutant field after noise reduction (DB5, 5-layer decomposition, custom threshold: $55.5 \%)$

is no way to change it, and the flexibility is low when processing large amounts of data. Therefore, this study used a custom threshold, which can be adjusted freely when the denoising effect is not good. A custom threshold provides more flexibility than a fixed threshold.

As discussed above, this study selected the DB5 wavelet to decompose the pollutant field in four layers, and the denoising effect was good when the custom threshold was above 55.5\% per level. As shown in Figures 17(b) and (c), when the three-layer decomposition was performed with a threshold of $55.5 \%$ for each level, there was obvious noise residue, and the fitting accuracy was not high. Although there was no noise during the fifth-order decomposition, there was considerable signal distortion. Therefore, a four-layer decomposition similar to the frequency of the characteristic signal of people's walking was ultimately chosen.

Finally, the temperature, flow and pollutant field after noise reduction were fitted with high precision through Gaussian function. It was worth noting that the impact on the final result mainly occurs in the denoising process. If distortion appeared, as shown in Figure 15(b), it would cause that the eigenvalues extracted by Gaussian function fitting deviated from the actual situation. If a large noise residue appeared, as shown in Figure 17(b), it would lead to the fact that the Gaussian function would not be able to accurately identify the location of the wave peaks and eventually lead to failure of the fitting. According to Nyquist sampling law and wavelet basis function selection method, these two situations should be avoided, so as to ensure the accuracy of Gaussian fitting results.

\section{Conclusion}

In this study, the method for quantitatively calculating the robustness of a ventilation system in response temperature, flow and pollutant field fluctuations was developed. The original experimental data was first denoised by FFT filtering or wavelet transform; was then fitted using a Gaussian function; the range and time disturbance feature was subsequently extracted; and finally the robustness was calculated by means of the developed dimensionless equations.

For the temperature field, the range scale and time scale robustness values for the CS system were the lowest, while the values measured for the CC, SC and SS systems were 
similar. For the flow field, the operation of the ventilation system increased the range scale robustness by $19.7 \%-39.4 \%$. The largest range scale robustness value was achieved by the SS system, and it was $16.5 \%$ higher than that exhibited by the worst ventilation case. For the pollutant field, the ventilation system increased the range scale robustness by $10.0 \%-38.8 \%$. The largest range scale robustness value was achieved by the SS system and was $25.7 \%$ higher than the smallest value. However, all the ventilation systems had a very limited effect in controlling the disturbance time for the flow field and pollutant fields.

The selection methods for FFT filtering and wavelet transform, FFT filter cut-off frequency, wavelet function, decomposition layers and threshold for wavelet denoising were also discussed. The calculation methods can be adjusted correspondingly if the human walking frequency or sampling frequency differs from that in other studies.

\section{Acknowledgements}

This study was supported by the National Natural Science Foundation of China (No. 52108075), Natural Science Foundation of Hebei Province, China (No. E2020202147), S\&T Program of Hebei (No. 216Z4502G), Fundamental Research Funds of Hebei University of Technology (No. JBKYTD2003) and Hebei Province Funding Project for Returned Scholars, China (No. C20190507).

\section{References}

Agirman A, Cetin YE, Avci M, et al. (2020). Effect of air exhaust location on surgical site particle distribution in an operating room. Building Simulation, 13: 979-988.

Bachman G, Narici L, Beckenstein E (2012). Fourier and Wavelet Analysis. New York: Springer.

Brohus H, Balling KD, Jeppesen D (2006). Influence of movements on contaminant transport in an operating room. Indoor Air, 16: 356-372.

Cao S-J, Cen D, Zhang W, et al. (2017). Study on the impacts of human walking on indoor particles dispersion using momentum theory method. Building and Environment, 126: 195-206.

Chang L, Tu S, Ye W, et al. (2017). Dynamic simulation of contaminant inleakage produced by human walking into control room. International Journal of Heat and Mass Transfer, 113: $1179-1188$

Dai H, Zhao B (2020). Association of the infection probability of COVID-19 with ventilation rates in confined spaces. Building Simulation, 13: 1321-1327.

Daubechies I (1992). Ten Lectures on Wavelets. Philadelphia, PA, USA: Society for Industrial and Applied Mathematics.

Deng N, Jiang CS (2012). Selection of optimal wavelet basis for signal denoising. In: Proceedings of the 9th International Conference on Fuzzy Systems and Knowledge Discovery, Chongqing, China.
Desai PS, Sawant N, Keene A (2021). On COVID-19-safety ranking of seats in intercontinental commercial aircrafts: A preliminary multiphysics computational perspective. Building Simulation, 14: $1585-1596$.

Dong Y, Zhu L, Li S, et al. (2021). Optimal design of building openings to reduce the risk of indoor respiratory epidemic infections. Building Simulation, https://doi.org/10.1007/s12273021-0842-3.

Gothwal H, Kedawat S, Kumar R (2011). Cardiac arrhythmias detection in an ECG beat signal using fast Fourier transform and artificial neural network. Journal of Biomedical Science and Engineering, 4: 289-296.

Guo H (2011). A simple algorithm for fitting a Gaussian function [DSP tips and tricks]. IEEE Signal Processing Magazine, 28: 134-137.

Hang J, Li Y, Jin R (2014). The influence of human walking on the flow and airborne transmission in a six-bed isolation room: Tracer gas simulation. Building and Environment, 77: 119-134.

He H, Wang Z, Tan Y (2015). Noise reduction of ECG signals through genetic optimized wavelet threshold filtering. In: Proceedings of IEEE International Conference on Computational Intelligence and Virtual Environments for Measurement Systems and Applications (CIVEMSA), Shenzhen, China.

Ji W, Chen, Zhao B (2021). A comparative study of the effects of ventilation-purification strategies on air quality and energy consumption in Beijing, China. Building Simulation, 14: 813-825.

John AM, Khanna K, Prasad RR, et al. (2020). A Review on Application of Fourier Transform in Image Restoration. In: Proceedings of the Fourth International Conference on I-SMAC (IoT in Social, Mobile, Analytics and Cloud) (I-SMAC), Palladam, India.

Kong X, Guo C, Lin Z, et al. (2021). Experimental study on the control effect of different ventilation systems on fine particles in a simulated hospital ward. Sustainable Cities and Society, 73: 103102.

Kumar A, Singh M (2015). Optimal selection of wavelet function and decomposition level for removal of ECG signal artifacts. Journal of Medical Imaging and Health Informatics, 5: 138-146.

Liu X, Han G (2018). Information-theoretic extensions of the ShannonNyquist sampling theorem. arXiv:1810.08089.

Liu Z, Liu H, Rong R, et al. (2020). Effect of a circulating nurse walking on airflow and bacteria-carrying particles in the operating room: An experimental and numerical study. Building and Environment, 186: 107315.

Liu T, Guo Y, Hao X, et al. (2021a). Evaluation of an innovative pediatric isolation (PI) bed using fluid dynamics simulation and aerosol isolation efficacy. Building Simulation, 14: 1543-1552.

Liu Z, Zhu H, Song Y, et al. (2021b). Quantitative distribution of human exhaled particles in a ventilation room. Building Simulation, https://doi.org/10.1007/s12273-021-0836-1.

Liu S, Zhao X, Nichols SR, et al. (2022). Evaluation of airborne particle exposure for riding elevators. Building and Environment, 207: 108543.

Lv L, Wu Y, Cao C, et al. (2021). Impact of different human walking patterns on flow and contaminant dispersion in residential kitchens: Dynamic simulation study. Building Simulation, https://doi.org/10.1007/s12273-021-0844-1. 
Mallat SG (1989). A theory for multiresolution signal decomposition: The wavelet representation. IEEE Transactions on Pattern Analysis and Machine Intelligence, 11: 674-693.

Massarotti N, Mauro A, Mohamed S, et al. (2021). Fluid dynamic and thermal comfort analysis in an actual operating room with unidirectional airflow system. Building Simulation, 14: 1127-1146.

Mazumdar S, Poussou SB, Lin CH, et al. (2011). Impact of scaling and body movement on contaminant transport in airliner cabins. Atmospheric Environment, 45: 6019-6028.

McKeen P, Liao Z (2022). The influence of airtightness on contaminant spread in MURBs in cold climates. Building Simulation, 15: 249-264.

Mei X, Zeng C, Gong G (2021). Predicting indoor particle dispersion under dynamic ventilation modes with high-order Markov chain model. Building Simulation, https://doi.org/10.1007/s12273-0210855-y.

Ngui WK, Leong MS, Hee LM, et al. (2013). Wavelet analysis: Mother wavelet selection methods. Applied Mechanics and Materials, 393: 953-958.

Paskaranandavadivel N, O'Grady G, Du P, et al. (2013). Comparison of filtering methods for extracellular gastric slow wave recordings. Neurogastroenterology and Motility, 25: 79-83.

Poussou SB, Mazumdar S, Plesniak MW, et al. (2010). Flow and contaminant transport in an airliner cabin induced by a moving body: Model experiments and CFD predictions. Atmospheric Environment, 44: 2830-2839.

Rao KR, Kim DN, Hwang JJ (2010). Fast Fourier Transform: Algorithms and Applications. Dordrecht, Netherlands: Springer.

Ren J, Wade M, Corsi RL, et al. (2020). Particulate matter in mechanically ventilated high school classrooms. Building and Environment, 184: 106986.

Ren J, He J, Kong X, et al. (2022a). A field study of $\mathrm{CO}_{2}$ and particulate matter characteristics during the transition season in the subway system in Tianjin, China. Energy and Buildings, 254: 111620.

Ren J, Tang M, Novoselac A (2022b). Experimental study to quantify airborne particle deposition onto and resuspension from clothing using a fluorescent-tracking method. Building and Environment, 209: 108580

Resnikoff HL, Wells RO Jr (1998). The mallat algorithm. Wavelet Analysis. New York: Springer

Ruikar SD, Doye DD (2011). Wavelet based image denoising technique. International Journal of Advanced Computer Science and Applications, 2(3): 49-53.

Satheesan MK, Mui KW, Wong LT (2020). A numerical study of ventilation strategies for infection risk mitigation in general inpatient wards. Building Simulation, 13: 887-896.

Shemi PM, Shareena EM (2016). Analysis of ECG signal denoising using discrete wavelet transform. In: Proceedings of IEEE
International Conference on Engineering and Technology (ICETECH), Coimbatore, India.

Sifuzzaman M, Islam M, Ali M (2009). Application of wavelet transform and its advantages compared to Fourier transform.

Srivastava S, Zhao X, Manay A, et al. (2021). Effective ventilation and air disinfection system for reducing coronavirus disease 2019 (COVID-19) infection risk in office buildings. Sustainable Cities and Society, 75: 103408.

Tao Y, Inthavong K, Tu JY (2017). Dynamic meshing modelling for particle resuspension caused by swinging manikin motion. Building and Environment, 123: 529-542.

Veer K, Agarwal R (2015). Wavelet and short-time Fourier transform comparison-based analysis of myoelectric signals. Journal of Applied Statistics, 42: 1591-1601.

Wang J, Chow TT (2011). Numerical investigation of influence of human walking on dispersion and deposition of expiratory droplets in airborne infection isolation room. Building and Environment, 46: 1993-2002.

Wang L, Xu L, Feng S, et al. (2013). Multi-Gaussian fitting for pulse waveform using Weighted Least Squares and multi-criteria decision making method. Computers in Biology and Medicine, 43: 1661-1672.

Wang H, Tian C, Wang W, et al. (2019). Temporal cross-correlations between ambient air pollutants and seasonality of tuberculosis: a time-series analysis. International Journal of Environmental Research and Public Health, 16: 1585.

Weisberg M (2006). Robustness analysis. Philosophy of Science, 73: $730-742$.

Whyte W, Whyte WM, Blake S, et al. (2013). Dispersion of microbes from floors when walking in ventilated rooms. International Journal of Ventilation, 12: 271-284.

Wu TX, Hua HX (2014). Mechanical Vibration. Beijing: Tsinghua University Press. (in Chinese)

Wu W, Lin Z (2015). Experimental study of the influence of a moving manikin on temperature profile and carbon dioxide distribution under three air distribution methods. Building and Environment, 87: 142-153.

Ye J, Qian H, Ma J, et al. (2021). Using air curtains to reduce short-range infection risk in consulting ward: A numerical investigation. Building Simulation, 14: 325-335.

Yin Y, He J, Zhao L, et al. (2022). Identification of key volatile organic compounds in aircraft cabins and associated inhalation health risks. Environment International, 158: 106999.

Zhang D (2019).Wavelet transform. In Fundamentals of Image Data Mining.

Zhao RM, Cui HM (2015). Improved threshold denoising method based on wavelet transform, In: Proceedings of the 7th International Conference on Modelling, Identification and Control (ICMIC), Sousse, Tunisia. 